\title{
The Effects of Inhomogeneities on Evaluating the Deceleration Parameter $\mathrm{q}_{0}$
}

\author{
R. Kantowski, T. Vaughan, and D. Branch Q \\ University of Oklahoma, Department of Physics and Astronomy, \\ Norman, OK 73019, USA
}

Received —

${ }^{1}$ Kantowski@phyast.nhn.uoknor.edu 


\begin{abstract}
Analytic expressions for distance-redshift relations which have been corrected for the effects of some inhomogeneities in the Friedmann-Robertson-Walker (FRW) mass density are used to illustrate the significance of inhomogeneities on a determination of $q_{0}$ made by using Type Ia supernovae. The value of $q_{0}$ inferred from a given set of observations depends on the fractional amount of matter in inhomogeneities and is up to 50 percent larger than that obtained by using the standard Mattig $m-z$ result for pure dust FRW models.
\end{abstract}

Subject headings: cosmology: theory - large-scale structure of universe 


\section{Introduction}

Some years ago one of us (Kantowski 1969, henceforth referred to as K1) used the "Swiss-cheese" models to study the effects of local inhomogeneities in the FRW mass density on the propagation of light through an otherwise homogeneous and isotropic universe. The Swiss-cheese models are standard pressure-free FRW models (sometimes called dust models) from which non-overlapping comoving spheres of zero-temperature material (called dust) have been removed and each replaced by a mass concentrated at its center. At a given epoch the model looks like Swiss-cheese full of perfectly round holes each with a seed (representing a spherical mass) at its center (see Fig. 1). If the holes are small compared to the radius of the FRW universe, the mass of the seed differs indistinguishably from the integrated mass of the dust missing from the sphere (see K1). The comoving spheres expand/contract right along with the universe itself. The gravity field inside each expanding sphere is given by a Schwarzschild metric of concentrated mass. The importance of Swiss-cheese models is that they are explicit exact solutions to GR (with all boundary conditions being satisfied). In

such models, effects of local inhomogeneities on observable relations can be calculated free of superposition approximations.

The large scale structure of the universe, i.e., which pressure free FRW model we are actually living in, is clearly unaffected by how the clumps are distributed in number density or size. The FRW structure is fixed by giving the Hubble $\left(H_{0}\right)$ and the deceleration $\left(q_{0}\right)$ parameters. However, as we will show, observational determination of $q_{0}$ is critically affected by the clumps. Most observations involve collecting radiation from a finite-sized distant source with a much smaller ( $\sim$ point $)$ receiver. The cross section of the radiation (the beam) ultimately received is consequently of the same order as the source's size. It is this size that is important in deciding what matter is to be ascribed to the seeds and what to the cheese. Matter whose size is small compared to the beam (e.g. an atom) is included in the cheese (the homogeneous dust) and matter whose size is large compared to the beam (e.g. a galaxy) is put into the seeds (the inhomogeneous clumps). The reader should remember that this is a model which is intended to mimic the optical effects of matter and not its actual physical distribution. For example atomic gas clouds are not physically confined away from galaxies as is the cheese; however, the optical effects of the gas should be independent of its location (see Section 2.). In this model matter the same size as the beam clearly causes trouble and has to be dealt with separately for each observation. The above separation of mass based on size is not completely well defined because of the changing beam size from source to receiver. Star clusters for example would be considered small near a galactic sized source but large near us.

In Section 2., optical propagation equations, originally derived in [K1, are restated. 
These equations describe the propagation of a beam of electromagnetic radiation traversing a clumpy universe. The reader is directed to $\mathbb{K} 1$ for details and to Sections 4.5.2 and 4.5.3 of Schneider et al. 1992 for a complete review of more recent work. In the Appendix it is concluded that the net effect of the seeds is negligible for $z<1$ (except for uncommon lensing type situations) and in Section 3. it is shown that the resulting $b=0$ propagation equation for the cross sectional area of the beam is the associated Legendre equation. ${ }^{2}$

The main result of this paper is the clear presentation of the corrected luminosity distance [expression (18) combined with (14)] and the clear demonstration of the necessity of its use when evaluating $q_{0}$. In Section 4 . the importance of taking into account the effects of inhomogeneities is demonstrated by showing how a recent determination of $q_{0}$ (made by applying the magnitude-redshift relation to SN1992bi) will be altered.

\section{Swiss-cheese Optics}

Observable relations such as magnitude-redshift or angular size-redshift are determined from expressions for the cross sectional area $A$ of a light beam that propagates between source and observer. In K1 an integral-differential equation for the average area $A$ as it propagates through a Swiss-cheese universe (see Fig. 1) was given as

$$
\frac{\sqrt{A}^{\prime \prime}}{\sqrt{A}}+\frac{\left\langle\xi^{2}\right\rangle}{A^{2}}=\frac{\rho_{D}}{\rho_{F}} \mathcal{R}_{F}
$$

where

$$
\begin{aligned}
\prime & \equiv \frac{d}{d v}=-\frac{H_{0}}{c}(1+z)^{3}\left(1+2 q_{0} z\right)^{1 / 2} \frac{d}{d z}, \\
\left\langle\xi^{2}\right\rangle & =\frac{\rho_{I}}{\rho_{F}} b^{3} \int_{0}^{v} A^{2}(1+z)^{6} d v
\end{aligned}
$$

\footnotetext{
${ }^{2}$ Before this manuscript could be completed and submitted and unbeknownst to us, equation (11) and its solutions (18) and (21) were published by Seitz \& Schneider 1994. They deserve credit for first recognizing (9) as the Associated Legendre equation and giving its general solution. They also show that the general solution reduces to previously known special solutions for appropriate parameter values. We give our version of the solution for completeness only. The conjectured extension of the validity of the $b=0$ equation beyond the Swiss-Cheese models is argued by Schneider et al. 1992 in Sections 4.5.2 and 4.5.3.
} 


$$
\begin{aligned}
\mathcal{R}_{F} & =-\frac{4 \pi G}{c^{2}} \rho_{F}(0)(1+z)^{5} \\
& =-3 q_{0} \frac{H_{0}^{2}}{c^{2}}(1+z)^{5}
\end{aligned}
$$

and where Weinberg sign conventions have been used (Misner et al. 1973). In (1), $\rho_{F}$ is the usual FRW mass density (the local mass density of the cheese) and in (舟) $\rho_{F}(0)$ is its current value. The other two mass densities, $\rho_{D}$ and $\rho_{I}$, are the components of $\rho_{F}$ made up of dust and inhomogeneities respectively, $\rho_{F}=\rho_{D}+\rho_{I}$. In the Swiss-cheese model $\rho_{I}$ is given by summing all clumped masses in a large volume (containing a representative number of clumps) and dividing by that volume. The density $\rho_{D}$ is similarly computed by summing only the dust mass. The two driving terms for the area $A$ in (1) are proportional to $\mathcal{R}_{F}$ and $\left\langle\xi^{2}\right\rangle$ which represent average converging effects on the beam as it passes respectively

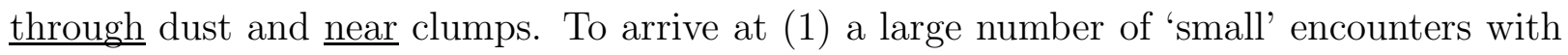
clumps had to be assumed. Any single encounter that causes a 'large' fractional change in the beam's observed cross sectional area was excluded. Such encounters cause lensing effects and must necessarily be accounted for by other means (Bourassa \& Kantowski 1975). The $v$ parameter is a particular affine parameter which has been expressed in (2) as a differential operator in redshift z. Equations (2), (3), and (1) are slightly simpler than the corresponding equations in $\mathbb{K} 1$ because of the choice of the particular affine parameter made here (note that no loss of physical generality is made by such a choice). The electromagnetic wave starts from a point on the source at $z=z_{s}$ where $v=0$, and expands at first as a small part of a spherical wave front, e.g. as a disk. As the spherical wave front encounters a clump the disk is distorted into an ellipse. The $\left\langle\xi^{2}\right\rangle$ term in (11) is the effect on the wave front's area caused by repeated distortions of the clumps. The parameter $b$ measures the strength of the distortions and has units of (length) ${ }^{-1}$. Its value is defined by

$$
b^{3}=12 q_{0} \frac{H_{0}^{2}}{c^{2}} \frac{\left\langle\left(G m / c^{2}\right)^{4 / 3}\right\rangle}{\left\langle\left(G m / c^{2}\right)\right\rangle}\left[\frac{\left(G m / c^{2}\right)^{1 / 3}}{a}\right]^{2},
$$

which makes $b$ the order of the cube root of the Schwarzschild radius of a typical clump divided by its opaque radius squared (i.e., by $a^{2}$ ) and by the Hubble radius of the universe squared (i.e., by $c^{2} / H_{0}^{2}$ ).

The reader is directed to K1 for details and assumptions made in all derivations, including the somewhat unphysical assumption that $m / a^{3}$ is the same for all clumps. If $b=0$, (11) is easily seen to be a linear homogeneous second-order equation in $\sqrt{A}$ [ i.e., see (9)]; however, if $b \neq 0$, upon differentiation, (四) becomes a linear homogeneous third-order equation in $A$, 


$$
A^{\prime \prime \prime}-4\left[\frac{\rho_{D}}{\rho_{F}} \mathcal{R}_{F}\right] A^{\prime}-2\left[\frac{\rho_{D}}{\rho_{F}} \mathcal{R}_{F}^{\prime}-\frac{\rho_{I}}{\rho_{F}} b^{3}(1+z)^{6}\right] A=0 .
$$

The area $A$ in (目) and (6), when evaluated at the observer, is related to the luminosity distance $D_{\ell}$ by $\delta \Omega D_{\ell}^{2}=\left.A\right|_{0}\left(1+z_{s}\right)^{2}$ or equivalently $\delta \theta D_{\ell}=\left.\sqrt{A}\right|_{0}\left(1+z_{s}\right)$. The luminosity distance is defined in terms of the flux received $\mathcal{F}$ and the power $\delta \mathcal{P}$ radiated into solid angle $\delta \Omega$ by $\mathcal{F}=\delta \mathcal{P} / \delta \Omega D_{\ell}^{2}$. The solid and plane angles are constants measured at the source $\left(z=z_{s} \leftrightarrow v=v_{s}=0\right)$ for a wave front expanding into the future. The initial conditions for (何) are $\left.\sqrt{A}\right|_{s}=0$ and $d \sqrt{A} /\left.d v\right|_{s}=\delta \theta\left(1+z_{s}\right)$ (or equivalently $d \sqrt{A} /\left.d z\right|_{s}=-\delta \theta c /\left[H_{0}\left(1+z_{s}\right)^{2} \sqrt{1+2 q_{0} z_{s}}\right)$. The additional boundary condition needed for (6) comes from (3) and is $d^{2} \sqrt{A} /\left.d v^{2}\right|_{s}=0$. These boundary conditions are a consequence of our choice of affine parameter $v$ in (2), so chosen to simplify "apparent size" boundary conditions (see the next section). The general power series solution with $b \neq 0$ is:

$$
\begin{aligned}
\left.\sqrt{A}\right|_{0} / \delta \theta & =D_{\ell} /(1+z)=\frac{c}{H_{0}}\left\{z-\frac{1}{2}\left[1+q_{0}\right] z^{2}+\frac{1}{2}\left[1+\frac{\rho_{I}}{\rho_{F}} q_{0}+q_{0}^{2}\right] z^{3}\right. \\
& \left.-\frac{1}{2}\left[1+\frac{\rho_{I}}{\rho_{F}} q_{0}-\left(\frac{1}{4}-\frac{3}{2} \frac{\rho_{I}}{\rho_{F}}\right) q_{0}^{2}+\frac{5}{4} q_{0}^{3}+\frac{1}{30} b^{3} \frac{c^{3}}{H_{0}^{3}} \frac{\rho_{I}}{\rho_{F}}\right] z^{4}+O\left[z^{5}\right]\right\},
\end{aligned}
$$

where the redshift $z$ is now the source's redshift. This result agrees with the Mattig result (Mattig 1958),

$$
D_{\ell}^{M}=r R_{0}(1+z)=\frac{c}{H_{0} q_{0}^{2}}\left\{q_{0} z+\left(q_{0}-1\right)\left(\sqrt{1+2 q_{0} z}-1\right)\right\}
$$

when $\rho_{I}=0$.

If $b$ can be neglected the difference between the standard Mattig result (8) and (7) [or the exact result (18)] is due to the diminished converging effect of the mass remaining in the beam, i.e., the $\left(\rho_{D} / \rho_{F}\right) \mathcal{R}_{F}$ term in (1). The standard result assumes all matter is uniformly distributed in and out of the beam. If some fraction $\rho_{I} / \rho_{F}$ exists only out of the beam where it acts only through a negligible $b^{3}$ term, then a diminished fraction $\rho_{D} / \rho_{F}$ remains to converge the beam. In the Appendix we conclude that for a large majority of observations the $b^{3}$ term can be neglected. We now explicitly give the analytic solution of (四) when $b=0$.

\section{The Analytic Solution For $A(z)$ when $b=0$}


The solution of (11) for $b=0$ and $\rho_{D}=0$ was given by Dyer \& Roeder 1972 and is the limiting case $\left(\rho_{D} \rightarrow 0\right)$ of result (18) below. We first start by rewriting (11) when $b=0$ as

$$
(1+z)^{3} \sqrt{1+2 q_{0} z} \frac{d}{d z}(1+z)^{3} \sqrt{1+2 q_{0} z} \frac{d}{d z} \sqrt{A(z)}+\frac{\rho_{D}}{\rho_{F}} 3 q_{0}(1+z)^{5} \sqrt{A(z)}=0 .
$$

This equation [3 $^{3}$ can next be put into a recognizable self-adjoint form by changing the independent variable from $z$ to $\zeta$ and the dependent variable from $\sqrt{A(z)}$ to $P(\zeta)$,

$$
\begin{aligned}
1+z & \equiv\left(1-\frac{1}{2 q_{0}}\right)\left(1-\zeta^{2}\right), \\
(1+z)^{2} \sqrt{A(z)} & \equiv\left(1-\zeta^{2}\right) P(\zeta) .
\end{aligned}
$$

For $q_{0}<1 / 2$ we have $\zeta^{2}>1$ and for $q_{0}>1 / 2, \zeta$ is imaginary. The resulting equation

$$
\left(1-\zeta^{2}\right) \frac{d^{2} P}{d \zeta^{2}}-2 \zeta \frac{d P}{d \zeta}+\left[6 \frac{\rho_{I}}{\rho_{F}}-\frac{4}{1-\zeta^{2}}\right] P=0,
$$

has solutions which are arbitrary linear combinations of the associated Legendre functions of the first and second kind (Abramowitz \& Stegun 1964) i.e.,

$$
P(\zeta)=c_{1} P_{\nu}^{2}(\zeta)+c_{2} Q_{\nu}^{2}(\zeta)
$$

where

$$
\nu \equiv\left(\sqrt{1+24 \rho_{I} / \rho_{F}}-1\right) / 2,
$$

is the positive root of $\nu(\nu+1)=6 \rho_{I} / \rho_{F}$. The range for $\nu$ is from $\nu=0$ (usual pure dust FRW) where no clumps are present, to $\nu=2$ where all mass is in the form of clumps and no dust is present.

Boundary conditions can now be chosen appropriate for either luminosity distance $D_{\ell}$ as above or for apparent-size/angular distance $D_{L}$. For apparent-size distances $\sqrt{A}=\delta \theta D_{L}$, the boundary values are specified at the observer, $\left.D_{L}\right|_{0}=0$ and $d D_{L} /\left.d v\right|_{0}=-1$ (or equivalently $d D_{L} /\left.d z\right|_{0}=c / H_{0}$ ). For the $b=0$ case, the two types of solutions are easily related by the Wronskian of (G) (see Etherington 1933),

$$
D_{\ell}(z)=D_{\llcorner}(z)(1+z)^{2} .
$$

${ }^{3}$ See the Appendix A of Seitz \& Schneider 1994 for the first presentation of this material including a comparison of all previously known limiting solutions. 
Now switch to the more easily implemented apparent-size boundary conditions and hence require:

$$
\begin{aligned}
\left.\left(c_{1} P_{\nu}^{2}(\zeta)+c_{2} Q_{\nu}^{2}(\zeta)\right)\right|_{z=0} & =0 \\
\left.\frac{q_{0}}{\sqrt{1-2 q_{0}}}\left(c_{1} \frac{d P_{\nu}^{2}(\zeta)}{d \zeta}+c_{2} \frac{d Q_{\nu}^{2}(\zeta)}{d \zeta}\right)\right|_{z=0} & =\frac{c}{H_{0}}
\end{aligned}
$$

These two equations can be used to find $c_{1}$ and $c_{2}$. The results then simplify by using the constancy of the Wronskian of (11),

$$
W\left[P_{\nu}^{2}(\zeta), Q_{\nu}^{2}(\zeta)\right] \equiv P_{\nu}^{2}(\zeta) \frac{d Q_{\nu}^{2}(\zeta)}{d \zeta}-Q_{\nu}^{2}(\zeta) \frac{d P_{\nu}^{2}(\zeta)}{d \zeta}=\frac{(\nu+2)(\nu+1)(\nu)(\nu-1)}{\left(1-\zeta^{2}\right)}
$$

to give

$$
\begin{aligned}
& c_{1}=\frac{c}{H_{0}} \frac{1}{\sqrt{1-2 q_{0}}} \frac{2}{(\nu+2)(\nu+1)(\nu)(\nu-1)} Q_{\nu}^{2}\left(\frac{1}{\sqrt{1-2 q_{0}}}\right), \\
& c_{2}=-\frac{c}{H_{0}} \frac{1}{\sqrt{1-2 q_{0}}} \frac{2}{(\nu+2)(\nu+1)(\nu)(\nu-1)} P_{\nu}^{2}\left(\frac{1}{\sqrt{1-2 q_{0}}}\right) .
\end{aligned}
$$

The resulting expression for apparent-size distance is

$$
\begin{aligned}
& D_{L}\left(q_{0}, \nu ; z\right)=\frac{c}{H_{0}} \frac{1}{\sqrt{1-2 q_{0}}} \frac{2}{(\nu+2)(\nu+1)(\nu)(\nu-1)} \times \\
& \left(\frac{1}{1+z}\right)\left[Q_{\nu}^{2}\left(\frac{1}{\sqrt{1-2 q_{0}}}\right) P_{\nu}^{2}\left(\frac{\sqrt{1+2 q_{0} z}}{\sqrt{1-2 q_{0}}}\right)-P_{\nu}^{2}\left(\frac{1}{\sqrt{1-2 q_{0}}}\right) Q_{\nu}^{2}\left(\frac{\sqrt{1+2 q_{0} z}}{\sqrt{1-2 q_{0}}}\right)\right] .
\end{aligned}
$$

This result is valid for all $q_{0} \geq 0$ but some care has to be taken with the limit $q_{0} \rightarrow 1 / 2$ as well as with the $\nu \rightarrow 0,1$ limits. The $\nu \rightarrow 0$ limit $\left(\rho_{I}=0\right)$ is the Mattig result for FRW and is related to (8) by (14). The $\nu=2$ (i.e., the $\rho_{D}=0$ Dyer \& Reoder 1972 result) is also obtained from (18) [see Seitz \& Schneider 1994].

Because the associated Legendre equation (11) is a special case of the hypergeometric equation the above solution (18) can be written in terms of hypergeometric functions. This makes the analytic continuation to $q_{0} \geq 1 / 2$ transparent and gives a resulting expression for $D_{L}\left(\right.$ or $\left.D_{\ell}\right)$ whose constituent parts no longer have singularities. Computer routines

\footnotetext{
${ }^{1}$ That differential equation (1) was of the hypergeometric type when $b=0$ was recognized
} by Dyer \& Roeder 1973. 
can then be used to evaluate these distances without having to avoid the singularities at $q_{0}=1 / 2, \nu=0$, and $\nu=1$. The two identities needed are:[

$$
\begin{aligned}
P_{\nu}^{2}(\zeta) & =\frac{\Gamma\left(-\frac{1}{2}-\nu\right) \zeta^{-\nu+1}}{2^{\nu+1} \pi^{1 / 2}\left(\zeta^{2}-1\right)}{ }_{2} F_{1}\left(\frac{\nu}{2}-\frac{1}{2}, \frac{\nu}{2} ; \nu+\frac{3}{2} ; \frac{1}{\zeta^{2}}\right) \\
& +\frac{2^{\nu} \Gamma\left(\frac{1}{2}+\nu\right) \zeta^{\nu+2}}{\pi^{1 / 2} \Gamma(\nu-1)\left(\zeta^{2}-1\right)}{ }_{2} F_{1}\left(-\frac{\nu}{2}-1,-\frac{\nu}{2}-\frac{1}{2} ; \frac{1}{2}-\nu ; \frac{1}{\zeta^{2}}\right), \\
Q_{\nu}^{2}(\zeta) & =\frac{\pi^{1 / 2} \Gamma(\nu+3) \zeta^{-\nu+1}}{2^{\nu+1} \Gamma\left(\nu+\frac{3}{2}\right)\left(\zeta^{2}-1\right)}{ }_{2} F_{1}\left(\frac{\nu}{2}-\frac{1}{2}, \frac{\nu}{2} ; \nu+\frac{3}{2} ; \frac{1}{\zeta^{2}}\right) \\
& =\frac{\pi^{1 / 2} \Gamma(\nu+3) \zeta^{-\nu-3}\left(\zeta^{2}-1\right)}{2^{\nu+1} \Gamma\left(\nu+\frac{3}{2}\right)}{ }_{2} F_{1}\left(\frac{\nu}{2}+2, \frac{\nu}{2}+\frac{3}{2} ; \nu+\frac{3}{2} ; \frac{1}{\zeta^{2}}\right),
\end{aligned}
$$

giving the second form of the solution to equation (9),

$$
\begin{aligned}
& D_{L}\left(q_{0}, \nu ; z\right)=\frac{c}{H_{0}} \frac{1}{\left(\nu+\frac{1}{2}\right)} \times \\
& {\left[\frac{\left(1+2 q_{0} z\right)^{1+\nu / 2}}{(1+z)^{2}}{ }_{2} F_{1}\left(\frac{\nu}{2}+2, \frac{\nu}{2}+\frac{3}{2} ; \nu+\frac{3}{2} ; 1-2 q_{0}\right){ }_{2} F_{1}\left(-\frac{\nu}{2}-1,-\frac{\nu}{2}-\frac{1}{2} ; \frac{1}{2}-\nu ; \frac{1-2 q_{0}}{1+2 q_{0} z}\right)\right.} \\
&-\left.\frac{1}{\left(1+2 q_{0} z\right)^{3 / 2+\nu / 2}}{ }_{2} F_{1}\left(-\frac{\nu}{2}-1,-\frac{\nu}{2}-\frac{1}{2} ; \frac{1}{2}-\nu ; 1-2 q_{0}\right){ }_{2} F_{1}\left(\frac{\nu}{2}+2, \frac{\nu}{2}+\frac{3}{2} ; \nu+\frac{3}{2} ; \frac{1-2 q_{0}}{1+2 q_{0} z}\right)\right] .
\end{aligned}
$$

This form is well behaved as a function of $\nu$ as well as in the neighborhood of $q_{0}=1 / 2$, and is needed beyond the circle of convergence $(|\xi|=1)$ of the hypergeometric series ${ }_{2} F_{1}(a, b ; c ; \xi)$ only on the negative real axis $\xi=x \leq-1$ when $q_{0} \geq 1$ (where it is easily continued). In Fig. 2, $D_{L}$ is plotted as a function of $z$ for three values of $\nu$ and three values of $q_{0}$ and in Fig. 3 the related magnitude-redshift curves are plotted. The reader should recall that apparent (bolometric) magnitudes $m$ are related to absolute (bolometric) magnitudes $M$ and luminosity distances $D_{\ell}$ [see (14)] by

$$
m=M+5 \log _{10}\left(D_{\ell} / 10 p c\right)=5 \log _{10}\left(\frac{H_{0}}{c} D_{\ell}\right)+\text { constant },
$$

which when evaluated as a function of redshift is called the $m-z$ (magnitude-redshift) relation. As pointed out in the next section the constant $\equiv M-5 \log _{10}\left(\frac{H_{0}}{c} 10 p c\right)$ turns out to be observationally independent of the Hubble parameter. In the next section we investigate the sensitivity of this relation to the clumpiness parameter $\nu$.

\footnotetext{
${ }^{2}$ Note that in 8.1.5 of Abramowitz \& Stegun 1964 a $\pi^{-1 / 2}$ is missing.
} 


\section{Application}

We conclude by illustrating the effect of inhomogeneities on the determination of $q_{0}$ when Type Ia supernovae (SNe Ia) are used in the Hubble diagram. Such a determination is expected to be feasible in the near future. Attempts to use galaxies or quasars in this way are foiled by the intrinsic dispersion in their luminosities and by uncertainties associated with their evolution. However, normal SNe Ia (those that have nonpeculiar spectra, light-curve shapes, and broad band colors) are luminous point sources that form a well defined ridge line in a Hubble diagram (for recent reviews of SNe Ia see Branch \& Khokhlov 1994 and references therein). Even just the application of a simple $\mathrm{B}-\mathrm{V}$ color criterion defines a subsample of $\mathrm{SNe}$ Ia that has mean absolute magnitudes $\mathrm{M}_{\mathrm{B}} \simeq \mathrm{M}_{\mathrm{V}} \simeq-19.8 \pm 0.1+5 \log \left(\mathrm{H}_{0} / 50\right)$ and dispersions about the means $\sigma\left(\mathrm{M}_{\mathrm{B}}\right) \simeq \sigma\left(\mathrm{M}_{\mathrm{V}}\right) \simeq 0.2$ magnitudes (Vaughan et al. 1995, Hamuy et al. 1995, Vaughan \& Branch 1995). With sufficient spectroscopic or photometric information it may be possible to control the individual SN Ia relative magnitudes to 0.1 or even better (Fisher et al. 1995, Hamuy et al. 1995, Riess, Press, \& Kirshner 1995, Tammann \& Sandage 1995). The value of $H_{0}$, and thus the calibration of the mean SN Ia absolute magnitude, does not enter the determination of $q_{0}$ from the Hubble diagram.

In the standard SN Ia model, a mass accreting white dwarf approaches the Chandraskhar limit, ignites degenerate carbon near its center, undergoes a thermonuclear instability, and disrupts completely. The light curve is powered by the radioactive decay of freshly synthesized ${ }^{56} \mathrm{Ni}$ through ${ }^{56} \mathrm{Co}$ to ${ }^{56} \mathrm{Fe}$. Thus, to first order, the peak absolute magnitude of SNe Ia is not expected to vary with cosmic time. Observationally, some evidence for a dependence of the properties of SNe Ia on the age of the stellar population at their sites has been found (Branch \& van den Bergh 1993, Hamuy et al. 1995). This constitutes evidence for mild evolution of the SN Ia population with cosmic time, but it implies that SNe Ia at high redshift, when the universe was younger, ought to have an even smaller dispersion among their properties than do the nearer SNe Ia that constitute the bulk of the present observational sample. In any case, with sufficient spectroscopic and photometric information it should be possible to identify counterparts of the high redshift SNe Ia among the nearer SNe Ia.

Recently it has been demonstrated that SNe Ia at cosmologically significant redshifts can be discovered and measured. Perlmutter et al. 1994 has announced the discovery of SN $1994 \mathrm{~F}$, a SN Ia at $z=0.354$; SN 1994G, a probable SN Ia at $z=0.425$; and SN 1994H, a probable SN Ia at $z=0.373$. Perlmutter et al. 1995 present a light curve of SN 1992bi, a probable SN Ia at $\mathrm{z}=0.458$, which they use with the standard Mattig relation to make a preliminary estimate of the value of $q_{0}$. Recently additional remote SNe Ia and probable 
SNe Ia have been discovered (C. Pennypacker, personal communication).

Fig. 4, which is an enlarged portion of Fig. 3 near the redshift $z=0.458$ of SN 1992bi, shows that clumping can have a significant effect on the determination of $q_{0}$ at this redshift. In Fig. 5, the quantity $5 \log _{10}\left(H_{0} / c\right) D_{l}\left(q_{0}, \nu ; z=0.458\right)$ [see (22)] is plotted as a function of $q_{0}$ for three values of the clumping parameter $\nu$. For example, if a given set of observations gives $q_{0}=0.1$ (Perlmutter et al. 1995) when the Mattig relation is used, the $\nu=2$ (completely clumpy, $\rho_{I}=\rho_{F}$ ) relation would give $q_{0}=0.15$; that is, if the universe is completely clumpy then neglecting to take clumpiness into account causes an underestimate of $q_{0}$ by about 50 percent. The difference between $q_{0}=0.1$ and $q_{0}=0.15$ is significant quantitatively, but not qualitatively. On the other hand, if the observations were such that the Mattig relation gave $q_{0}=0.34$, then the $\nu=2$ relation would give $q_{0}=0.51$. This difference is more than merely quantitative - it is the difference between an open and a closed universe.

We find that it is just when redshifts are large enough for the determination of $q_{0}$ that the effects of clumps become significant. Figs. 4 and 5 show that if $q_{0}$ were known,

distinguishing between a completely clumpy universe and a universe with no clumps would require that the average apparent magnitude of a set of remote $\mathrm{SNe}$ Ia be compared to that of nearby SNe Ia to an accuracy $\delta m \simeq 0.05$ magnitude. Such statistical accuracy should soon be available at the rate SNe Ia are being discovered. In practice, though, the $m-z$ relation only constrains the $q_{0}$ and $\nu$ parameters in combination. Independent information must be used to determine their individual values.

This work was supported by the Department of Energy, the Southern Association for High Energy Physics (SAHEP) funded by the Texas National Research Laboratory Commission (TNRLC), and by NSF grant AST 91-15061.

\section{A. Can the parameter $b$ be neglected?}

Based on the series solution (7), K1 concluded that the $b^{3}$ term appearing in (1) and (6) would be insignificant for observations of large objects such as galaxies. Further arguments are now given to reassert that the probability of the $b^{3}$ term affecting an observation is rather small. A priori, observing a significant effect of $b$ on the magnitude-redshift relation should be about as likely as a single observation resulting in a gravitational lens effect. When either lensing or a significant distortion of a light beam occurs, the cause is the 
same conformal curvature term in the gravity field. Rauch (1991) has come to a similar conclusion about the unlikely probability of observing gravitationally lensed SNe Ia at small $\mathrm{z}$ as we do here. In order to estimate the significance of focusing by external mass clumps on a passing beam of light, i.e., to estimate the relative significance of $b^{3}$ in (6), a comparison of the magnitudes of the two terms that appear as coefficients of $A$ can be made. Even if such a comparison implies that the $b^{3}$ term is significant compared to the $\mathcal{R}^{\prime}$ term, they both might be unimportant compared to the $z$ development caused by the $A^{\prime \prime \prime}$ or $A^{\prime}$ terms. The ratio of the two $A$ terms is

$$
\frac{\rho_{I}}{\rho_{D}} \frac{b^{3}(1+z)^{6}}{\mathcal{R}^{\prime}}=\frac{\rho_{I}}{\rho_{D}}\left(\frac{b^{3}}{15 q_{0}\left(H_{0} / c\right)^{3}}\right) \frac{1}{(1+z) \sqrt{1+2 q_{0} z}} .
$$

The unitless combination

$$
B_{0} \equiv \frac{b^{3}}{15 q_{0}\left(H_{0} / c\right)^{3}}=\frac{4}{5} \frac{c}{H_{0}} \frac{\left\langle\left(G m / c^{2}\right)^{4 / 3}\right\rangle}{\left\langle\left(G m / c^{2}\right)\right\rangle}\left[\frac{\left(G m / c^{2}\right)^{1 / 3}}{a}\right]^{2},
$$

is clearly the parameter determined by the structure of the inhomogeneities, which affects the relative importance. In the series solution (7), $B_{0}$ first appears in the $z^{4}$ term, already indicating that it is probably not important at small redshifts.

Dyer \& Roeder 1974 numerically integrated (6) for numerous values of $\rho_{I} / \rho_{D}$ and $B \equiv 15 B_{0}$. From their graphs we can conclude that as long as $B_{0} \sim 1$ and observations are at redshifts $z<1$ then the $b^{3}$ term is not significant. In Fig. 6 the recomputed numerical solutions of (6) are given for values of $B_{0}=0,1$, and $5, q_{0}=0.1$ and 0.5 , and $\rho_{I} / \rho_{F}=0$ and 1 , confirming the above conclusion. For example for $q_{0}=0.5$ the magnitude of a SN Ia at $z \approx 0.5$ in the standard dust filled FRW universe would appear 0.075 magnitudes brighter than in the $B_{0}=0$ clumpy universe. If, in the clumpy universe, $B_{0}=1$ or $B_{0}=5$ the magnitude would be brighter by 0.01 and 0.05 magnitudes respectively. For $q_{0}=0.1$ all changes are about $1 / 4$ of those for $q_{0}=0.5$. The conclusion is that for $B_{0} \sim 1$ (and smaller) the $b^{3}$ term makes only a small increase in intensity $(\sim 1 / 7$ or less $)$ compared to the decrease in intensity due to the missing matter in the light beam $(\nu=2$ rather than $\nu=0$ ). As to what $B_{0}$ values are known to exist: for globular clusters, $B_{0} \sim 10^{-2}$; for galaxies and galaxy clusters, $B_{0} \sim 1$; for stars, $B_{0} \sim 10^{9}$; and for black holes at the centers of spiral galaxies, $B_{0} \sim 10^{25}$.

What is now shown is that the probability of seeing the effects of a $B_{0}$ larger than $\sim 1$ is quite small (for these limited redshifts). If it is assumed that there is a randomly distributed population of essentially point inhomogeneities of number density $n=n_{0} R_{0}^{3} / R^{3}$ between us and an observed object at redshift $z$, the probability that the observed light beam comes no closer than a distance $a^{\prime}$ to the center of any of the intervening masses can 
be computed by evaluating

$$
P(r)=\exp \left\{-\int_{0}^{r} \frac{n \pi a^{\prime 2} R d r}{\sqrt{1-k r^{2}}}\right\} .
$$

Assuming the clumps are points will maximize their possible effects. The parameter $r$ used here is the FRW comoving radial coordinate centered on the source and $R$ is the FRW scale factor. This result follows from the simple assumption that $-\Delta P \equiv P(r)-P(r+\Delta r)$, the probability a beam make it to $r$ having had no impact parameter less than $a^{\prime}$ and then having an impact parameter less than $a^{\prime}$ within the next $\Delta r$, is given by

$$
-\Delta P=P(r) \frac{\pi a^{\prime 2} \times n 4 \pi r^{2} \Delta r R^{3}}{4 \pi r^{2} R^{2} \sqrt{1-k r^{2}}} .
$$

This is a simple ratio of the sum of impact disk areas in a spherical shell of thickness $R \Delta r / \sqrt{1-k r^{2}}$ to the total area on the sky, at comoving distance $r$. After changing from co-moving coordinate $r$ to redshift $z$, (A3) can be easily integrated to give

$$
P(z)=\exp \left\{-\frac{\pi a^{\prime 2} n_{0} c}{H_{0}} f\left(z, q_{0}\right)\right\}
$$

where

$$
f\left(z, q_{0}\right)=\frac{1}{2 q_{0}^{2}}\left\{\frac{\left[\left(1+2 q_{0} z\right)^{3 / 2}-1\right]}{3}-\left(1-2 q_{0}\right)\left[\left(1+2 q_{0} z\right)^{1 / 2}-1\right]\right\} .
$$

If it is assumed that all members of this population of inhomogeneities have the same mass $m$, (A5) can be rewritten as a function of the unitless parameter $B_{0}^{\prime}$ of (A2) as

$$
P(z)=\exp \left\{-\frac{3 q_{0}}{5 B_{0}^{\prime}} \frac{\rho_{I}}{\rho_{F}} f\left(z, q_{0}\right)\right\} .
$$

$P(z)$ is interpreted as the probability that all impact parameters ( $a$ values as the light beam interacts with inhomogeneities on its path from a source at $z$ to us) have values such that $4 G m /\left(5 H_{0} c a^{2}\right)=B_{0}$ is less than $B_{0}^{\prime}$. In Fig. $7, P(z)$ is plotted for several values of $q_{0}$ and $\left(\rho_{I} / \rho_{F}\right) B_{0}^{\prime}$. For a given $B_{0}^{\prime}$ the maximum probability occurs when $\rho_{I} / \rho_{F}=1$, i.e., when all matter is in point clumps. From Fig. 7 it is clear that for redshifts $z<1$, the probability of having a value of $B_{0}<1$ is high. For such values of $B_{0}$ it has already been concluded that the $b$ term in (6) has a rather small effect. What is expected to happen is that an occasional observation of a SN Ia will occur where the intensity is increased because it happens to be closely aligned with a dark and unseen interveining galaxy. According to Fig. 7 this can't happen frequently. However, when it does, the SN will either sit far from the $m-z$ curve where it can be excluded, or sit close to the $m$ - $z$ curve and simply increase the error in the estimated value of $q_{0}$. 


\section{REFERENCES}

Abramowitz, M., \& Stegun, I. A. 1964, Handbook of Mathematical Functions, (New York: Dover Publications), 332 and 556

Bourassa, R. R., \& Kantowski, R. 1975, ApJ 195, 13

Branch, D., \& Khokhlov, A. M. 1995, Phys. Reports, in press

Branch, D., \& van den Bergh, S. 1993, AJ, 105, 2231

Dashevskii, V. M., \& Slysh, V. I. 1966, Soviet Astr.-AJ, 9, 671

Dyer, C. C., \& Roeder, R. C. 1972, ApJ 174, L115

Dyer, C. C., \& Roeder, R. C. 1973, ApJ 180, L31

Dyer, C. C., \& Roeder, R. C. 1974, ApJ 189, 167

Etherington, I. M. H. 1933, Phil. Mag. 15, 761

Fisher, A., Branch, D., Höeflich, P., \& Khokhlov, A. 1995, ApJ, submitted

Hamuy, M., Phillips, M. M., Maza, J., Suntzeff, N. B., Schommer, R. A., \& Aviles, R. 1995, AJ, in press

Kantowski, R. 1969, ApJ 155, 89 (see references)

Mattig, W. 1958, Astro. Nach. 284, 109

Misner, C. W., Thorne, K. S., \& Wheeler, J. A. 1973 Gravitation (San Francisco: W. H. Freeman)

Perlmutter, S. et al. 1995, ApJ, in press

Perlmutter, S., et al. 1994, I.A.U. Circular No 5956

Rauch, K. P. 1991, ApJ 374, 83

Riess, A., Press, W., \& Kirshner, R. P. 1995, ApJ, in press

Seitz, S., \& Schneider, P. 1994, A\&A 287, 349

Schneider, P., Ehlers, J., \& Falco, E. E. 1992, Gravitational Lenses (Berlin: Springer-Verlag) (see references and generalizations) 
Tammann, G. A., \& Sandage, A. 1995, ApJ, submitted

Vaughan, T. E, Branch, D., Miller, D. L., \& Perlmutter, S. 1995, ApJ, in press

Vaughan, T. E, \& Branch, D. 1995, in preparation 
Fig. 1. - Radiation beam of cross section $A$ propagating through a Swiss-cheese universe from distant source to observer.

Fig. 2.- Apparent-size distance $\frac{H_{0}}{c} D_{<}\left(q_{0}, \nu ; z\right)$ as a function of redshift $z$ for three values of $q_{0}$ and three values of $\nu$.

Fig. 3.- Magnitude-redshift relation, $5 \log _{10} \frac{H_{0}}{c} D_{\ell}\left(q_{0}, \nu ; z\right)$, as a function of redshift $z$ for three values of $q_{0}$ and three values of $\nu$.

Fig. 4.- Magnitude-redshift relation, $5 \log _{10} \frac{H_{0}}{c} D_{\ell}\left(q_{0}, \nu ; z\right)$, as a function of redshift $z$ for three values of $q_{0}$ and three values of $\nu$.

Fig. 5.- Magnitude-redshift relation, $5 \log _{10} \frac{H_{0}}{c} D_{\ell}\left(q_{0}, \nu ; z=0.458\right)$, as a function of the deceleration parameter $q_{0}$ for three values of $\nu$.

Fig. 6.- Magnitude-redshift relation, $5 \log _{10} \frac{H_{0}}{c} D_{\ell}\left(q_{0}, \nu, B_{0} ; z\right)$, as a function of redshift $z$ near 0.458 for two values of $q_{0}$ and $\nu$, and three values of $B_{0}$.

Fig. 7.- Probability of $B_{0}<B_{0}^{\prime}$ in a universe of given $\rho_{I} / \rho_{D}$ 


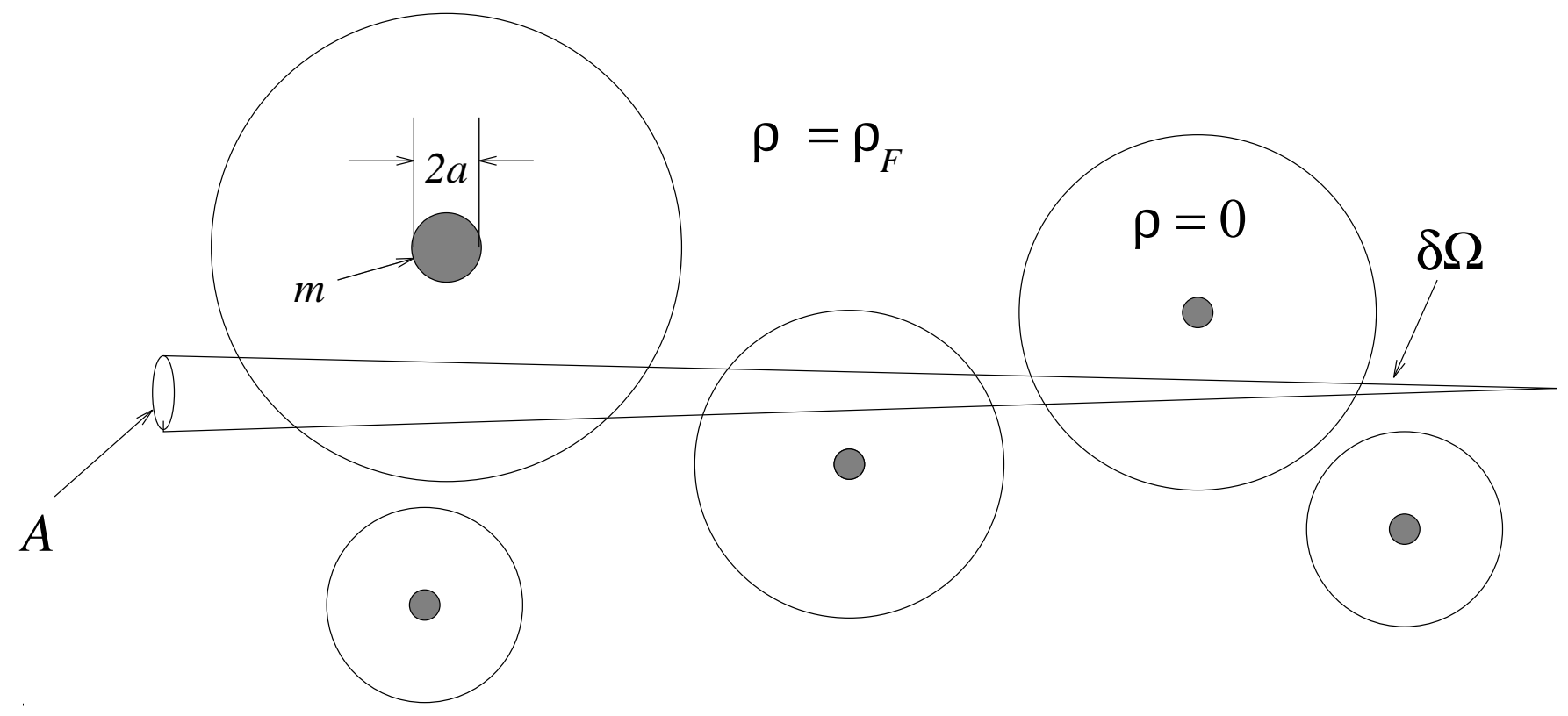

Fig. 1.- Radiation beam of cross section $A$ propagating through a swisscheese universe from distant source to observer. 


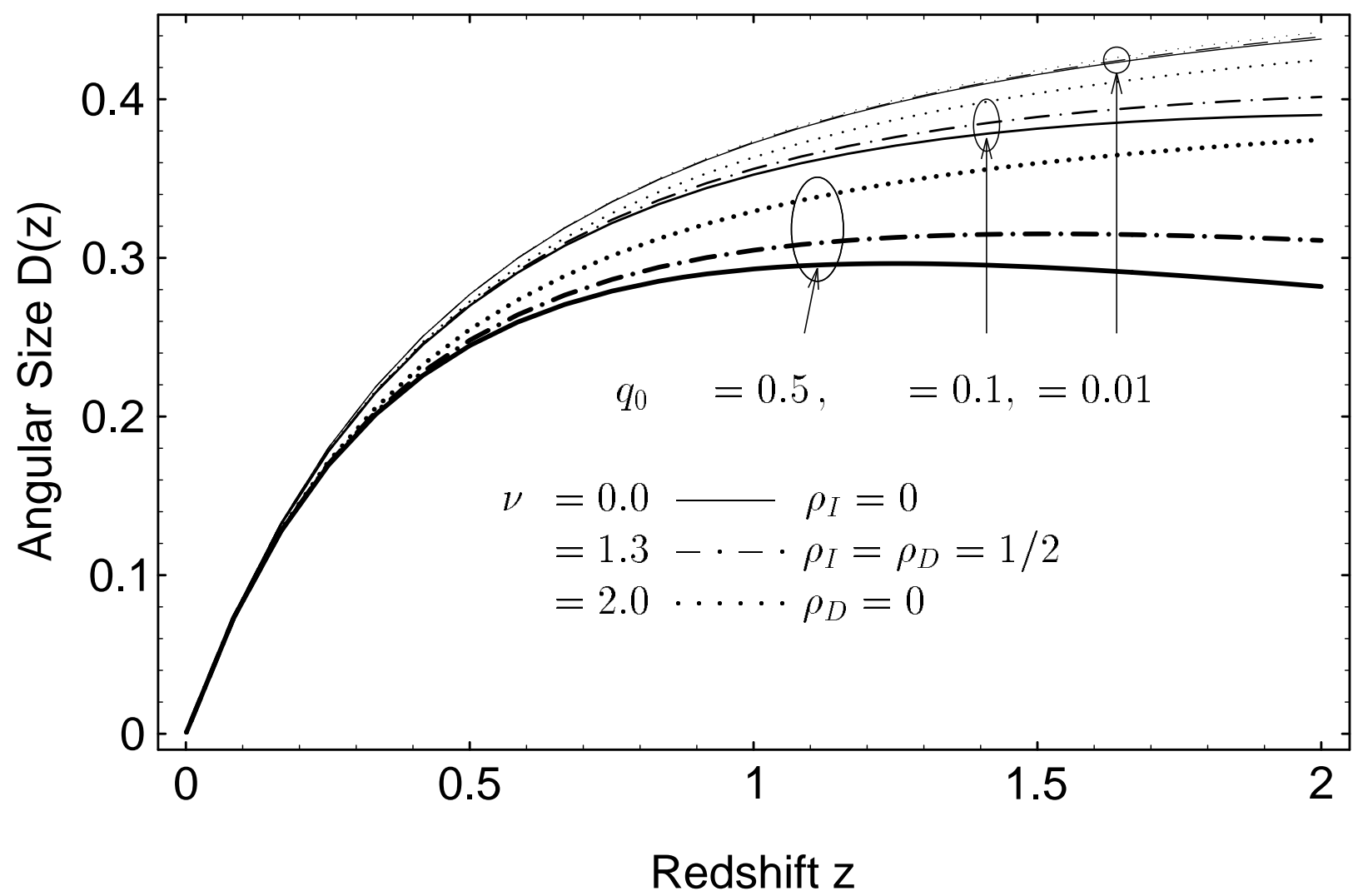

Fig. 2.- Apparent-size distance $\frac{H_{0}}{c} D_{L}\left(q_{0}, \nu ; z\right)$ as a function of redshift $z$ for three values of $q_{0}$ and three values of $\nu$. 


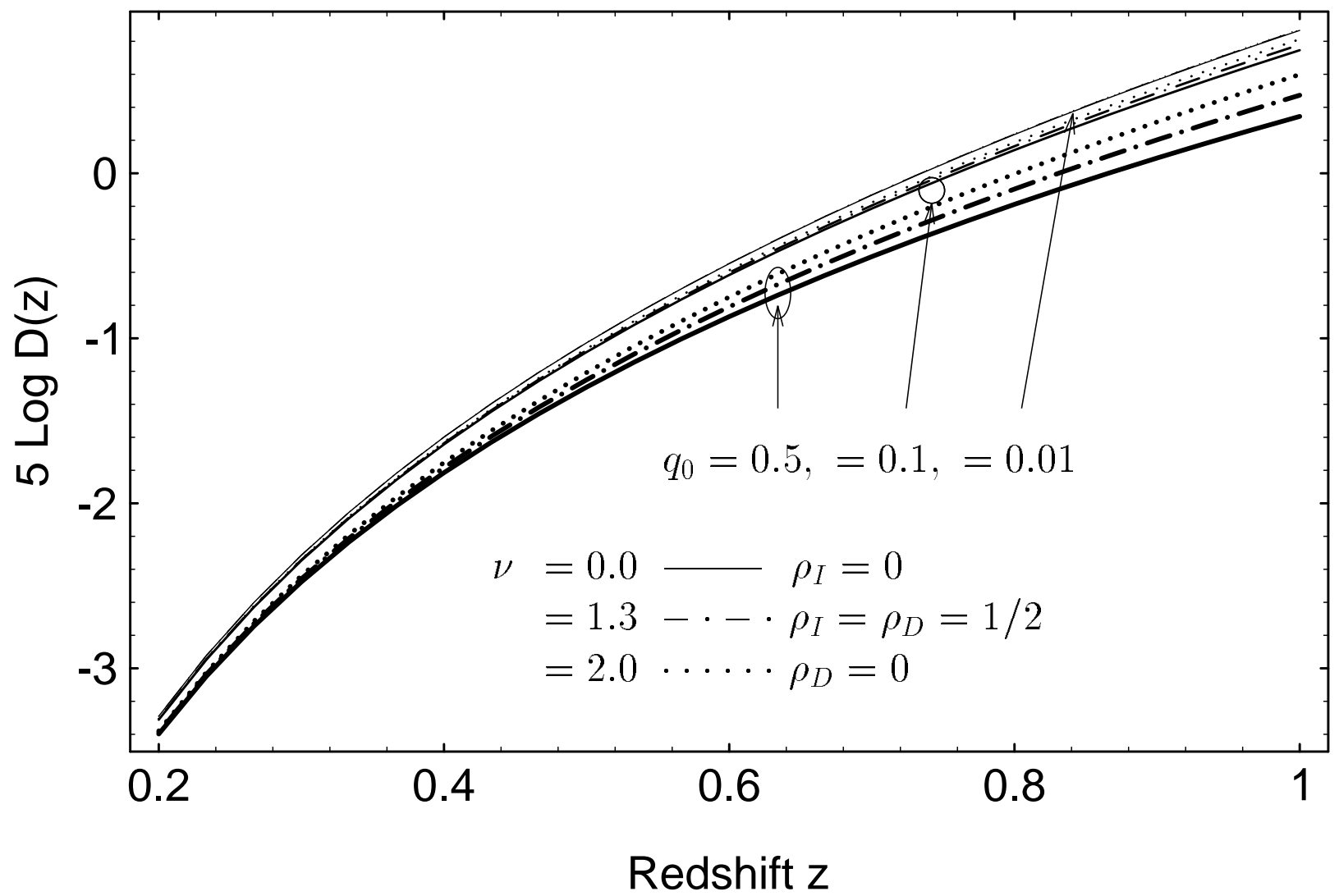

Fig. 3.- Magnitude-redshift relation, $5 \log _{10} \frac{H_{0}}{c} D_{\ell}\left(q_{0}, \nu ; z\right)$, as a function of redshift $z$ for three values of $q_{0}$ and three values of $\nu$. 


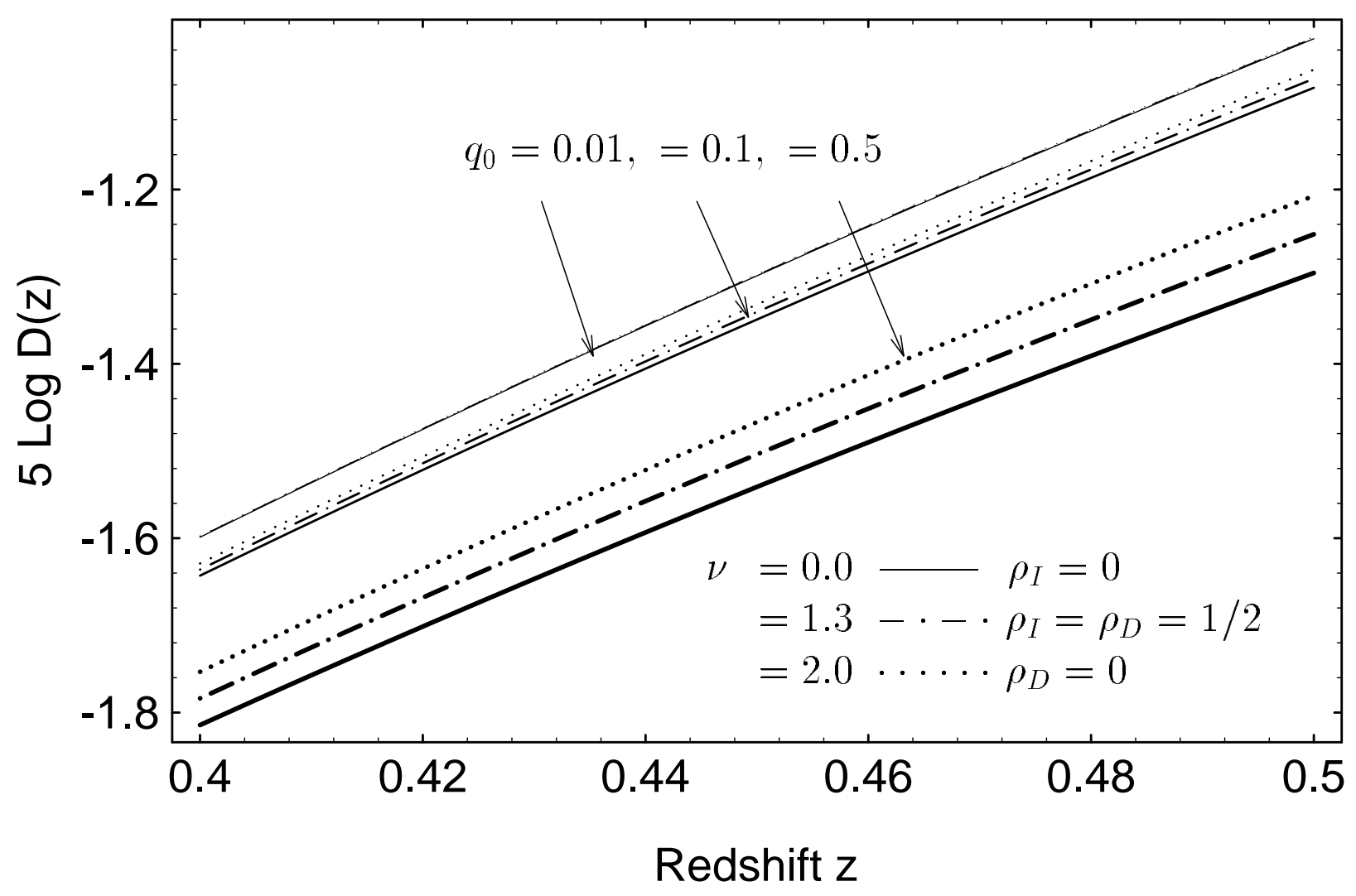

Fig. 4.- Magnitude-redshift relation, $5 \log _{10} \frac{H_{0}}{c} D_{\ell}\left(q_{0}, \nu ; z\right)$, as a function of redshift $z$ for three values of $q_{0}$ and three values of $\nu$. 


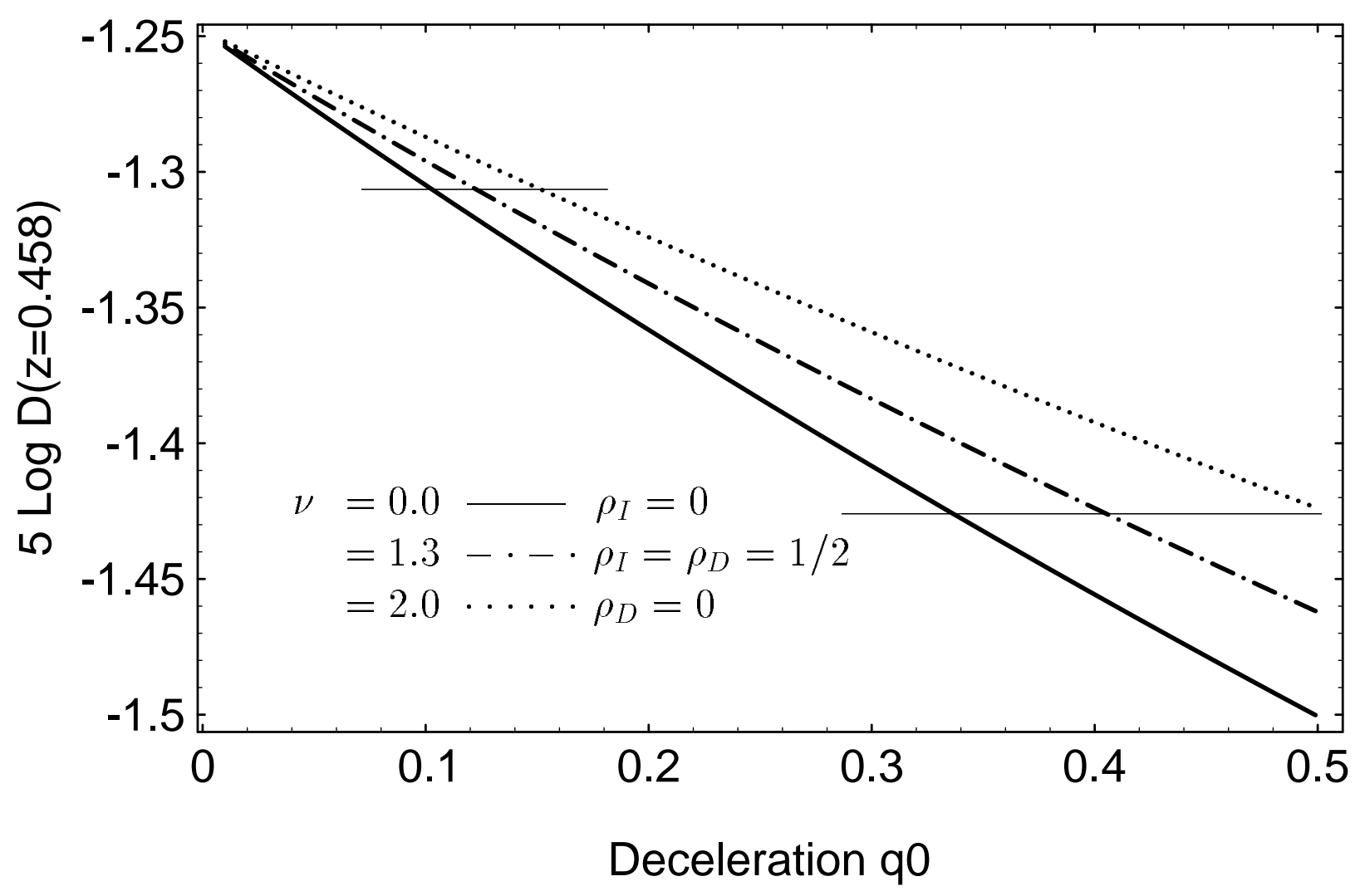

Fig. 5.- Magnitude-redshift relation, $5 \log _{10} \frac{H_{0}}{c} D_{\ell}\left(q_{0}, \nu ; z=0.458\right)$, as a function of the deceleration parameter $q_{0}$ for three values of $\nu$. 


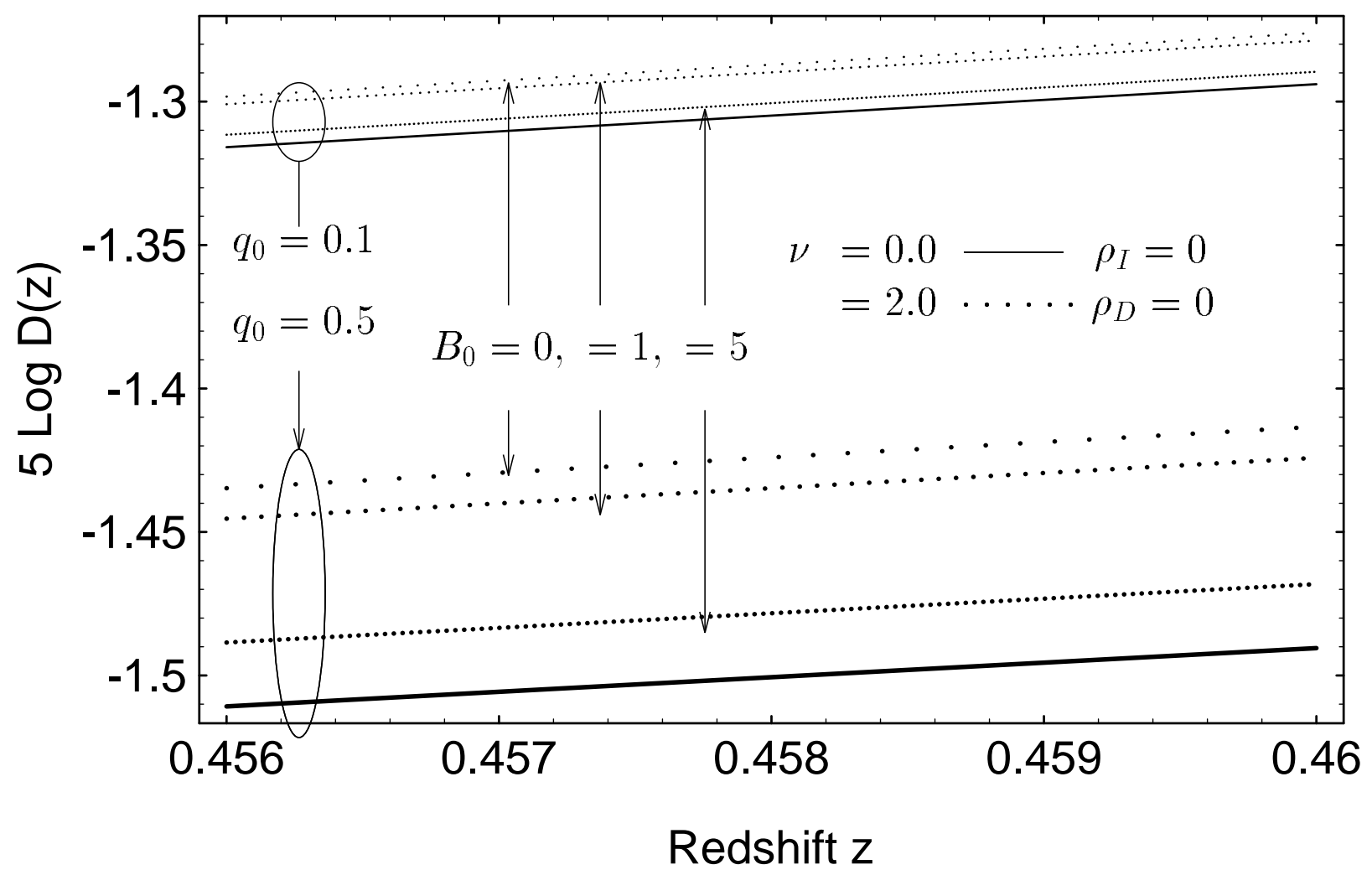

Fig. 6.- Magnitude-redshift relation, $5 \log _{10} \frac{H_{0}}{c} D_{\ell}\left(q_{0}, \nu, B_{0} ; z\right)$, as a function of redshift $z$ near 0.458 for two values of $q_{0}$ and $\nu$, and three values of $B_{0}$. 


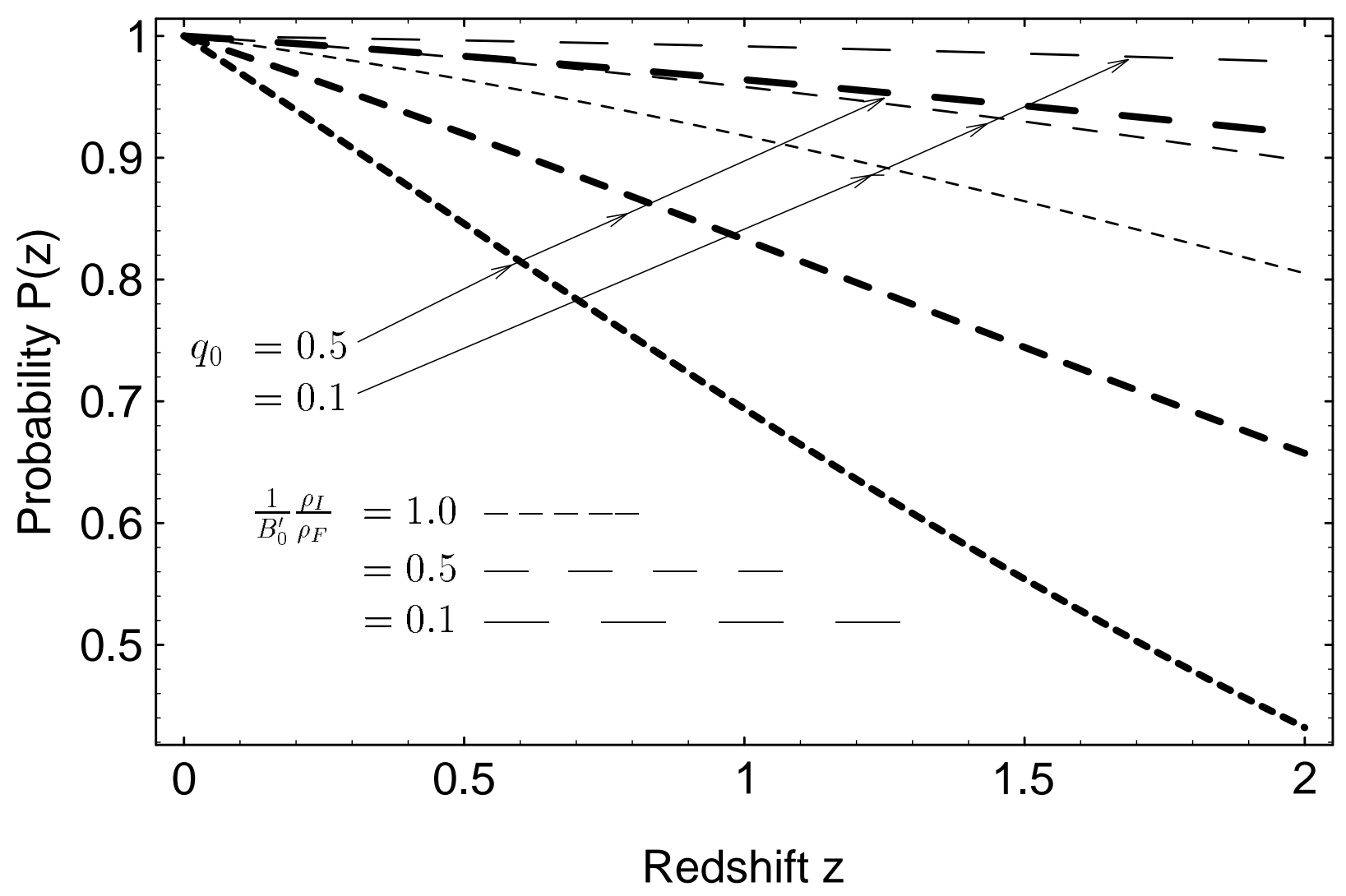

Fig. 7.- Probability of $B_{0}<B_{0}^{\prime}$ in a universe of given $\rho_{I} / \rho_{D}$ 